\title{
Cistectomía radical laparoscópica: Experiencia inicial
}

\author{
Núñez Mora C, García Mediero JMª , Cáceres Jiménez F, Cabrera Castillo PM \\ Centro Oncológico MD Anderson Internacional España. Madrid.
}

Actas Urol Esp. 2007;31(8):845-849

\section{RESUMEN}

CISTECTOMÍA RADICAL LAPAROSCÓPICA: EXPERIENCIA INICIAL

Objetivo: Presentar la experiencia inicial de nuestro grupo en cistectomía radical laparoscópica.

Pacientes y métodos: Entre septiembre de 2004 y junio de 2006 hemos realizado 16 cistectomías radicales laparoscópicas, 14 en varones y 2 en mujeres, con una edad media fue de 63,8 años (rango 51-85). Se realizaron 12 neovejigas ileales (anastomosis uretro-neovesical laparoscópica), 3 ureteroileostomías cutáneas y 1 ureterostomía cutánea. El seguimiento medio fue de 12,4 meses.

Resultados: La duración media de la cirugía fue de 340 minutos. La hemorragia media fue de $350 \mathrm{ml}$, requiriendo 3 pacientes transfusión intraoperatoria. La estancia hospitalaria media fue de 7,6 días. Se extrajeron una media de 22,9 ganglios, presentando metástasis ganglionares 6 de los 16 pacientes. La complicación más frecuente fue íleo paralítico en dos casos. No se han producido recidivas locales ni implantes en los puntos de inserción de los trócares.

Conclusiones: La cistectomía radical laparoscópica es una técnica compleja, con un alto tiempo quirúrgico que se ve compensado por la menor tasa de transfusión y la menor estancia hospitalaria. Los resultados oncológicos iniciales son superponibles a los de la cirugía abierta.

Palabras clave: Cistectomía radical. Laparoscopia. Cáncer vesical.

\section{ABSTRACT}

\section{LAPAROSCOPIC RADICAL CYSTECTOMY: INITIAL EXPERIENCE}

Objective: To review our initial experience with laparoscopic radical cystectomy.

Patients and methods.- Between September 2004 and June 2006 we performed 16 laparoscopic radical cystectomies (14 males and 2 females) with a median age of 63.8 y.o. (51-85). 12 ileal neobladder (with laparoscopic ileal-urethra anastomosis), 3 cutaneous ureteroileostomies and 1 cutaneous ureterostomy were performed as derivation techniques. Median follow up was 12.4 months

Resoults: Mean operation time was 340 minutes. Estimated blood loss was 350ml. and 3 cases required intra-op blood transfusión. Mean hospitalization discharged was at 7.6 days. Median linph node dissection was 22.9 finding node metastasis in 6 cases. Most frequent complication was ileo in two cases. No local recurrentes in trocar placement was achieved.

Conclusions: Laparoscopic Radical cystectomy is a challenged long-lasting procedure but with the advantage of a less transfusion rate and short hospital stay. Oncologycal outcomes are similar as tose from open surgery. 
$\mathrm{L}$ a cistectomía radical por vía abierta constituye actualmente el tratamiento de elección para los tumores de vejiga infiltrantes o superficiales refractarios a otros tratamientos ${ }^{1}$.

Aunque la realización de cistectomía radical por vía laparoscópica ya fue publicada en España por Sánchez de Badajoz en $1993^{2}$, la utilización de esta técnica de forma habitual ha tenido que esperar al desarrollo de otras técnicas laparoscópicas complejas ${ }^{3-5}$, que han permitido obtener una amplia destreza laparoscópica al tiempo que han demostrado una baja morbilidad.

En este artículo presentamos nuestra serie inicial de cistectomía radical laparoscópica.

\section{PACIENTES Y MÉTODOS}

Entre septiembre de 2004 y junio de 2006 se han realizado en nuestro Servicio 16 cistectomías radicales laparoscópicas. 14 pacientes eran varones y 2 mujeres. La edad media fue de 63,8 años (rango 51-85), con un índice de masa corporal medio de 27,1 (rango 20,8-33,1).

La indicación de la cistectomía fue tumor infiltrante en 13 casos y en 3 casos carcinoma vesical superficial de alto grado no controlable con BCG (Tabla 1). 2 pacientes tenían antecedentes de nefroureterectomía laparoscópica izquierda por tumores pielocaliciales (pT3aG3 en un caso y pT1mG3 en otro). Un paciente con un carcinoma microcítico de vejiga infiltrante recibió quimioterapia neoadyuvante.

Tabla 1. Indicaciones para la cistectomía radical

\begin{tabular}{lc}
\hline Carcinoma superficial multirrecidivante & 3 \\
Carcinoma urotelial infiltrante & 11 \\
Adenocarcinoma infiltrante & 1 \\
Carcinoma microcítico infiltrante & 1 \\
TOTAL & 16
\end{tabular}

En todos los casos se realizó cistoprostatectomía radical o exenteración pelviana (Fig. 1) (en mujeres) (Tabla 2), seguida en todos los casos de linfadenectomía laparoscópica (Fig. 2) incluyendo las cadenas ganglionares hasta la bifurcación aórtica. El extremo proximal de la uretra se cerró con hem-o-lok antes de la sección uretral para evitar diseminación de células tumorales. En un paciente se realizó simultáneamente nefroureterectomía derecha laparoscópica por anulación funcional.

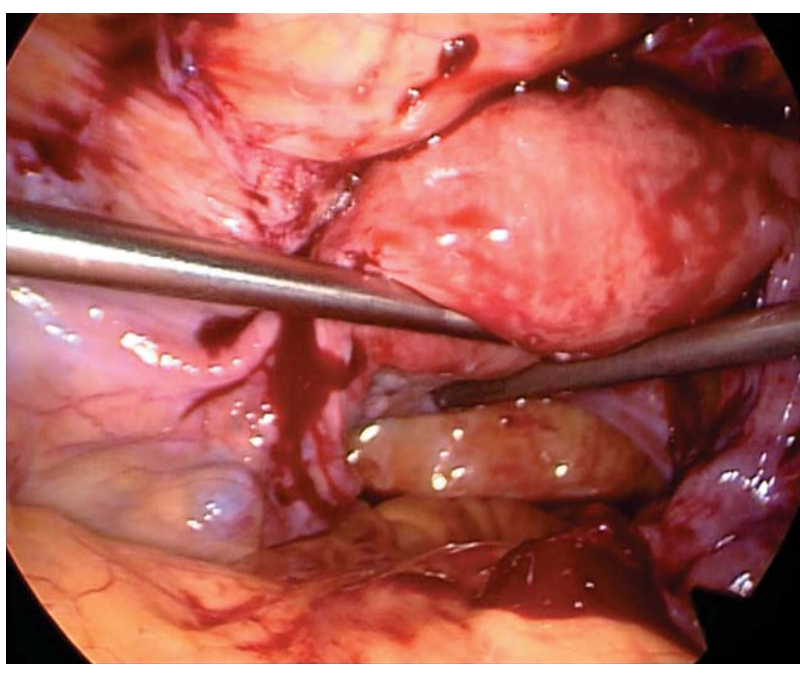

FIGURA 1. Exenteración anterior laparoscópica en una mujer.

Tabla 2. Técnica quirúrgica utilizada en la exéresis

Cistectomía radical en varón

Exenteración pelviana anterior

Cistectomía radical en mujer con

preservación vaginal

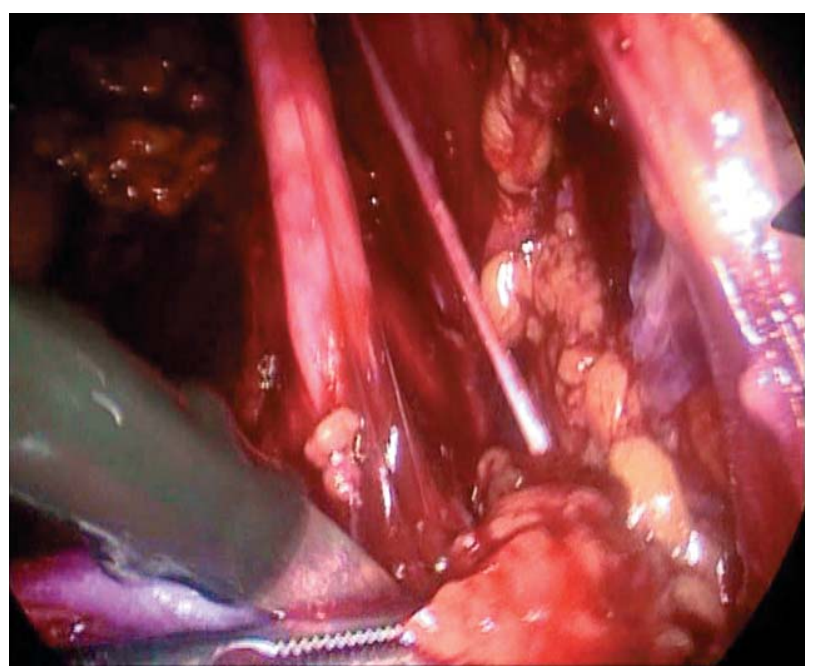

FIGURA 2. Linfadenectomía pelviana laparoscópica, consiguiendo una media de 22,9 ganglios por paciente.

En aquellos pacientes en los que se realizó ureteroíleostomía cutánea el uréter izquierdo se pasó hacia la derecha por vía laparoscópica a través de un túnel retroperitoneal, realizándose la placa de Wallace II de forma laparoscópica. Las piezas, una vez embolsadas se extrajeron por una incisión media de unos 6-7 cm de longitud, a través de la cual se exteriorizó el íleon y se realizó el 
tiempo intestinal de la derivación y las anastomosis urétero-intestinales. La anastomosis uretro-neovesical se realizó con una sutura continua con dos hebras ${ }^{6}$, asistida por laparoscopia en el primer caso y de forma laparoscópica pura en los demás.

Se realizaron 12 neovejigas ileales tipo Hautmann, 7 de ellas modificadas (Tabla 3), 3 ureteroileostomías cutáneas tipo Bricker-Wallace II (una de ellas en monorrena izquierda) y una ureterostomía cutánea derecha en un paciente monorreno.

Tabla 3. Tipo de derivación urinaria realizada

\begin{tabular}{lc}
\hline Neovejiga tipo Hautmann & 5 \\
Hautmann con chimenea ileal bilateral & 1 \\
Hautmann con chimenea ileal izquierda & 6 \\
Ureteroileostomía cutánea & 3 (1 monorreno) \\
Ureterostomía cutánea & 1 (monorreno) \\
\hline
\end{tabular}

Todos los uréteres se dejaron intubados, En las 4 derivaciones externas y en las primeras 7 neovejigas se utilizaron catéteres ureterales externos $6 \mathrm{Ch}$. En las últimas cinco neovejigas se utilizaron catéteres doble-J que se retiraron a las 6 semanas de la cirugía o al finalizar la quimioterapia adyuvante, en su caso.

El seguimiento medio fue de 12,4 meses (rango 4-25).

\section{RESULTADOS}

La duración media de la cirugía fue de 340 minutos (rango 210-480). La hemorragia media fue de $350 \mathrm{ml}$ (rango 100-1000 ml), requiriendo en 3 pacientes transfusión intraoperatoria . Un paciente requirió transfusión preoperatoria por hematuria.

La sonda nasogástrica se retiró en 13 casos a las 24 horas y en 3 a las 48 horas. Se inició tolerancia a las 24 horas en 11 casos y a las 48 horas en 3 . Hubo 2 cuadros de íleo paralítico que no permitieron iniciar la ingesta oral hasta los 3 y 4 días respectivamente.

La estancia media fue de 7,6 días (rango 5-11) en los casos de neovejiga con catéter externo ( 7 casos) fue de 8,2 días (rango 7-11), mientras que con pig-tail fue de 7 días (rango 5-11).

Como complicaciones postoperatorias inmediatas, aparecieron hemorragia pelviana autoli- mitada que requirió transfusión en 1 caso, seroma de herida en 1 y los mencionados 2 casos de íleo paralítico.

Los resultados histológicos de las piezas están resumidos en la Tabla 4.

Se extrajeron una media de 22,9 ganglios (1535) siendo positivos en 6 casos, 4 pN1 y 2 pN2 (Tabla 4). En 1 caso, los ganglios iliacos comunes fueron positivos (pM1). Los márgenes quirúrgicos fueron negativos en todos los casos.

Tabla 4. Resultado ánatomo-patológico de las piezas de cistectomía.

\begin{tabular}{lll}
\hline Categoria T & & \\
\hline & pTis-pT1G3 & 5 (2 alrededor de cicatriz \\
& & de RTU) \\
& pT2 & 1 \\
& pT3 & 7 \\
& pT4a & 1 \\
& pT2+pTis & 2 (Tis peritumoral) \\
\hline Categoria $\mathbf{N}$ & & \\
\hline & pNO & 11 \\
& pN1 & 2 (1 pT2b+pTis, 1 pT3b) \\
& pN2 & 2 (1 microcítico, 1 pM1+) \\
& pN3 & 1 (adenocarcinoma)
\end{tabular}

Con un seguimiento medio de 12,4 meses (rango 4-25), se ha producido una estenosis uretrovesical que requirió cervicotomía a los 7 meses. No se han producido estenosis uréterointestinales en los Bricker. En las neovejigas hemos tenido 3 estenosis de uréter izquierdo, solucionadas con dilatación endoscópica y cateterismo permanente (todos los pacientes $\mathrm{pN}+$ ) y 1 de uréter derecho sometido a reimplantación urétero-intestinal laparoscópica al ser infructuoso el cateterismo anterógrado.

Respecto a la evolución oncológica, aunque el seguimiento es corto, un paciente (adenocarcinoma pN3) falleció a los 20 meses de la cirugía por un accidente cerebrovascular isquémico sin signos de recidiva tumorarl. 4 pacientes (microcítico pN2, 1 pN1, 1 pN2, 1 pT3b) presentaron progresión de la enfermedad con metástasis a distancia. El resto de pacientes se encuentran libres de enfermedad. No se han producido recidivas locales ni implantes en los puertos. 


\section{DISCUSIÓN}

La difusión de la cistectomía radical laparoscópica se ha visto retrasada, respecto a otras técnicas laparoscópicas, por varios factores, tales como el riesgo de diseminación tumoral o de implantes en los trocares ${ }^{4,7} \mathrm{y}$ a la complejidad técnica que conlleva un elevado tiempo quirúrgico en una población habitualmente con comorbilidad importante. El desarrollo de la prostatectomía radical laparoscópica ha sido crucial para avanzar en el tratamiento laparoscópico de los tumores pélvicos ${ }^{3}$, y de hecho nosotros no comenzamos a realizar cistectomías hasta que llevábamos más de 150 prostatectomías radicales laparoscópicas.

Los beneficios del abordaje laparoscópico se traducen en un menor íleo paralítico y una disminución del dolor postoperatorio ${ }^{8,9}$. En nuestra serie hemos iniciado tanto la ingesta como la movilización de los pacientes de una forma lo más precoz posible con unos resultados óptimos respecto a la evolución postoperatoria.

Un beneficio añadido a la laparoscopia pelviana es la disminución de la hemorragia intraoperatoria y de la tasa de transfusión ${ }^{10-12}$. En la cistectomía radical laparoscópica se han publicado, incluyendo nuestra serie, tasas de transfusión de entre el 5 y el $24 \%^{10,11,13}$, inferiores a las publicadas con cirugía abierta ${ }^{14}$.

Desde el punto de vista teórico los posibles riesgos oncológicos de la cistectomía radical laparoscópica se encuentran en tres aspectos: la posibilidad de tener márgenes positivos; la posibilidad de diseminación peritoneal o de implantes en los trocares y, por último, la dificultad de realizar una linfadenectomía adecuada.

En nuestra serie no hemos tenido márgenes positivos ni recurrencias locales, a pesar de tratarse en 8 de los 16 casos de tumores localmente avanzados (pT3b, pT4). En algunas series de laparoscopia se dan cifras de márgenes positivos de entre el 0 y el 9\%11,13,15,16. En la cistectomía radical laparoscópica siempre se debe intentar conseguir un margen de seguridad libre de tumor y si existieran dudas de que la exéresis esté siendo completa consideramos preferible realizar una reconversión electiva a cirugia abierta que correr el riesgo de dejar un margen positivo.
Para evitar la diseminación intraperitoneal a través de la orina contenida en la vejiga realizamos un cierre completo del cabo proximal de la uretra mediante hem-o-lok previamente a la sección uretral, embolsando inmediatamente la pieza de cistectomía. Respecto a los implantes en los trocares, hasta el momento sólo se ha descrito un caso en una cistectomía robótica ${ }^{17}$. Para evitarlos aparte de impedir la salida de orina de la vejiga se deben evitar fugas de gas entre los trocares y la pared, así como realizar un embolsado inmediato de todas las piezas ${ }^{4}$.

La extensión de la linfadenectomía en la cistectomía radical ha demostrado tener valor pronóstico y terapéutico por lo que se tiende a realizar linfadenectomías extensas ${ }^{18-22}$, por lo que se considera correcta una linfadenectomía con un mínimo de entre 10 y 14 ganglios extraídos ${ }^{23}$. En el abordaje laparoscópico se debe realizar una linfadenectomía con los mismos límites que en cirugía abierta. Cuando nosotros comenzamos a realizar cistectomías radicales teníamos ya una amplia experiencia en prostatectomías radicales en las que realizamos habitualmente linfadenectomías, lo que nos permitió desde el principio alcanzar un número adecuado de ganglios extraídos, y esto a pesar de remitir conjuntamente todos los paquetes ganglionares (salvo los iliacos comunes).

Algunos grupos ${ }^{11,13}$ refieren en sus series iniciales la realización de linfadenectomías insuficientes, advirtiendo que con la experiencia aumenta el número de ganglios remitidos. Esta actuación nos parece cuestionable ya que, en general, lleva aparejada un riesgo de reducción de la supervivencia para el paciente ${ }^{20,22}$. Consideramos que si no se está seguro de conseguir una linfadenectomía adecuada sería mejor para el paciente completarla durante la fase de cirugia abierta.

Es evidente que la cistectomía radical laparoscópica conlleva, al menos inicialmente, un incremento del tiempo quirúrgico que, en nuestra serie, se ve claramente compensado por el menor íleo postoperatorio, el mejor control del dolor y la movilización efectiva precoz (deambulación).

Hemos tenido una estenosis urétero-intestinal derecha en el único caso de Hautmann modificado con chimenea ileal bilateral. Como se trata de 
un único caso no es posible evaluar el impacto del segmento ileal antiperistáltico en la etiología de dicha estenosis. Respecto al uréter izquierdo las 3 estenosis han ocurrido en pacientes con neovejigas tipo Hautmann clásicas $^{24}$. Desde que hemos recurrido a la realización de una chimenea ileal izquierda no han vuelto a producirse estenosis urétero-intestinales izquierdas. Esta modificación ha demostrado, en nuestra serie ser eficaz y no aumentar el tiempo quirúrgico, por lo que no nos planteamos el realizar las anastomosis urétero-intestinales por vía laparoscópica, con las que se han publicado tiempos quirúrgicos de hasta 11 horas $^{13}$, lo que probablemente explique en esas series la escasa o nula mejoría de los resultados postoperatorios respecto a la cirugía abierta.

\section{CONCLUSIONES}

La cistectomía radical laparoscópica es una técnica compleja que requiere una amplia experiencia laparoscópica previa. El incremento del tiempo quirúrgico se ve compensado por los beneficios en cuanto al menor íleo paralítico, menor dolor postoperatorio y movilización efectiva precoz. Los resultados oncológicos son superponibles a los de la cirugia abierta. Recomendamos realizar el tiempo intestinal y las anastomosis urétero-intestinales mediante minilaparotomía.

\section{REFERENCIAS}

1. Stein JP. Contemporary concepts of radical cystectomy and the treatment of bladder cancer. J Urol. 2003;169 (1):116117.

2. Sánchez de Badajoz E, Gallego Perales JL, Reche Rosado A, Gutierrez de la Cruz JM, Jimenez Garrido A. Cistectomía radical y conducto ileal laparoscópico. Arch Esp Urol. 1993; 46(7):621-624

3. Guilleneau B, Vallancien G. Laparoscopic radical prostatectomy: the Montsouris Technique. J Urol. 2000;163(6): 1643- 1649.

4. Rassweiler J, Tsivian A, Kumar AV, Lymberakis C, Schulze M, Seeman O et al.: Oncological safety of laparoscopic surgery for urological malignancy: experience with more than 1000 operations. J Urol. 2003;169(6):2072-2075.

5. Núñez Mora C, Cansino Alcaide R, Alonso Gregorio S, Martínez-Piñeiro Lorenzo L, de la Peña Barthel J; Enterocistoplastia de ampliación laparoscópica: experiencia inicial. Actas Urol Esp. 2007;31(1):17-22.

6. Van Velthoven RF, Ahlering TE, Peltier A, Skarecky DW, Clayman RV: Technique for laparoscopic running urethrovesical anastomosis: the single know method. Urology. 2003;61(4):699-702.

7. Tsivian A, Ami Sidi A. Port site metastases in urological laparoscopic surgery. J Urol. 2003;169(4):1213-1218.
8. Gill IS, Clayman RV, Mc Dougall EM. Advances in urological laparoscopy. J Urol. 1995;154(4):1275-1294.

9. Garrard Cl, Clements RH, Nanney L, Davidson JM, Richard WO. Adhesion formation after laparoscopic surgery. Surg Endosc. 1999;13(1):10-13.

10. Cathelineau X, Arroyo C, Rozet F, Barret E, Vallancien G. Laparoscopic assisted radical cystectomy: the Montsouris experience after 84 cases. Eur Urol. 2005;47(6):780-784.

11. Haber GP, Colombo JR, Aron M, Ukimura O, Gill IS. Laparoscopic radical cystectomy and urinary diversion: status in 2006. Eur Urol suppl. 2006;5(19):950-955.

12. Guilleneau B, el -Fettouh H, Baumert H, Cathelineau X, Doublet JD, Fromont G et al. Laparoscopic radical prostatectomy. oncological evaluation after 1000 cases a Montsouris Institute. J Urol. 2003;169(4):1261-1266.

13. Cansino JR, Cisneros J, Alonso S, Martínez-Piñeiro L, Aguilera A, Tabernero A et al. Laparoscopic radical cystectomy: initial series and analysis of results. Eur Urol suppl. 2006;5(19):956-961.

14. Novotny V, Hakenberg OW, Wiessner D, Heberling U, Litz $\mathrm{RJ}$, Oehlschlaeger $\mathrm{S}$ et al. Perioperative complications of radical cystectomy in a contemporary series. Eur Urol. 2007;51(2):397-402.

15. Gerullis H, Kuemmel C, Popken G. Laparoscopic cystectomy with extracorporeal-assisted urinary diversion: experience with 34 patients. Eur Urol. 2007;51(1):193-198.

16. Hemal AK, Kumar R, Seth A, Gupta NP. Complications of laparoscopic radical cystectomy during the initial experience. Int J Urol. 2004;1 1(7):483-488.

17. El-Tabey NA, Shoma AM: Port site metastases after robotassisted laparoscopic radical cystecyomy. Urology. 2005; 66(5): 1110.

18. Kassouf W, Leibovici D, Munsell MF, Dinney CP, Grossman $\mathrm{HB}$, Kamat AM. Evaluation of the relevance of lymph node density in a contemporary series of patients undergoing radical cystectomy. J Urol. 2006;176(1):53-57.

19. Herr HW. Superiority of ratio based lymph node staging for bladder cancer. J Urol. 2003;169(3):943-945.

20. Konety BR, Joslyn SA, O’Donnell MA. Extent of pelvic lymphadenectomy and its impact on outcome in patients diagnosed with bladder cancer : analysis of data from the surveillance, epidemology and end results program data base. J Urol. 2003;169(3):946-950.

21. Stein JP, Skinner DG. The role of lymphadenectomy in high-grade invasive bladder cancer. Urol Clin North Am. 2005;32(2): 187-197.

22. Stein JP, Lieskovsky G, Cote R, Groshen S, Feng AC, Boyd $\mathrm{S}$ et al. Radical cystectomy in the treatment of invasive bladder cancer: long-term results in 1054 patients. J Clin Oncol. 2001;19(3):666-675.

23. Monzó JL, Herranz Amo F, Cabello Benavente R, Hernández Fernández C. Utilidad de la linfadenectomía en el cáncer de vejiga. Revisión de la literatura. Actas Urol Esp. 2007;31(1):91-96.

24. Wenderoth UK, Bachor R, Eghart G, Frohneberg D, Miller K, Hautmann RE. The ileal neobladder: experience and results of more than 100 consecutive cases. J Urol. 1990; 143(3):492-496.

Correspondencia autor: Dr. C. Núñez Mora

Centro Oncológico MD Anderson Internacional España. Madrid.

Gómez Hemans, 2. 28033 Madrid. Tel.: 917878600

E-mail autor: cnunez@mdanderson.es

Información artículo: Original - Laparoscopia

Trabajo recibido: abril 2007

Trabajo aceptado: junio 2007 